\title{
Improved Classification Performance of Support Vector Machine Technique Using the Genetic Algorithm
}

\author{
Omar Saber Qasim \\ omar.saber@uomosul.edu.iq \\ Mohammed Ali Mohammed \\ College of Computer Science and Mathematics
}

University of Mosul, Mosul, Iraq

Received: $25 / 04 / 2018$

Accepted: 27/08/2018

\begin{abstract}
In this research, the genetic algorithm was proposed as a method to find the parameters of support vector machine, specifically the $\sigma$ and $\mathrm{c}$ parameters for kernel and the hyperplane respectively. Based on the Least squares method, the fitness function was built in the genetic algorithm to find the optimal values of the parameters in the proposed method. The proposed method showed better and more efficient results than the classical method of support vector machine which adopts the default or random values of parameters $\sigma$ and $\mathrm{c}$ in the classification of leukemia data .
\end{abstract}

Keywords: Genetic Algorithm; Support Vector Machine; Parameter Selection.

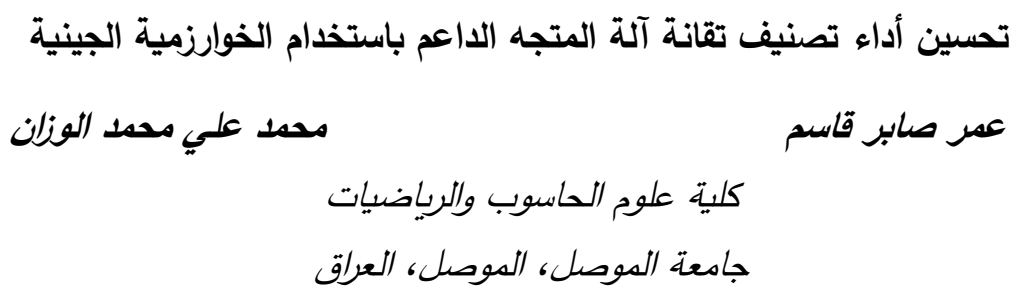

تاريخ قبول البحث: 2018108127

تاريخ استلام البحث: 2018104125

\section{(الملخص}

اقترح في هذا البحث الخوارزمية الجينية(Genetic algorithm) طريقة لإيجاد المعلمات Parameters)) لتقانة آلة المتجه الداعم (Support vector machine) وتحديدا المعلمات ه و c اللتان تمثلان على التوالي معلمة النواة (Kernel)، ومعلمة المستوى الفاصل Hyperplane))، وبالاستتاد إلى طريقة المربعات الصغرى (( Least)

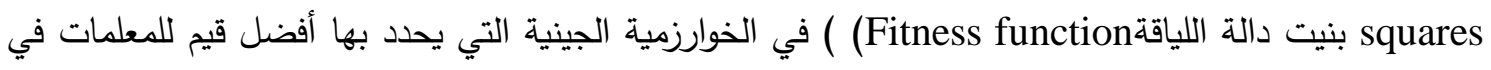
الطريقة المقترحة، وبالمقارنة مع الطريقة الاعتيادية لتقانة آلة المتجه الداعم التي تعتمد قيم افتراضية أو عشوائية

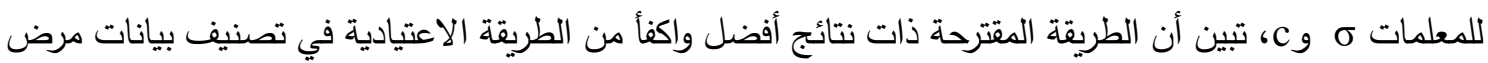
اللوكيميا. الكلمات المفتاحية: الخوارزمية الجينية، آلة المتجه الداعم، اختيار المعلمات. 1 تعد تقانات الذكاء الاصطناعي Techniques artificial intelligence من أهم الطرائق المستخدمة في العديد من المجالات (التطبيقات) وفي مقدمتها المجال الطبي. إذ تمتلك مرونـة في التعامل مع هذه التطبيقات

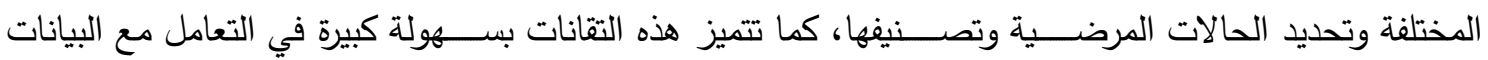


وتحليلها وتثخيصها بعدة إجراءات رياضية تعتمد على خوارزميات مبنية وفق نماذج رياضية متعددة. إذ إن العديد من التطبيقات وخصـوصــاً التطبيقات الطبية التي تعتمد التثـخيص الجزيئي Diagnosis molecular للجينات تحتوي كميات كبيرة من البيانات تصـل إلى الآف القراءات اللازمة لكل حالة مرضـية مما يؤدي إلى صـعوبة كبيرة في التعرف عليها من دون الأدوات والخوارزميات الحاسوبية التي تقوم بهذه المهمة. إن العديد من التقانـات الذكائيـة مثل الخوارزميـة الجينية Genetic algorithm وآلـة المتجـه الداعم Parameters وغيرها تعتمد على معلمات Fuzzy logic والمنطق المضـــــ Suport vector machine أسـاسية تدخل في تكوين هذه التقانات وتؤثر على نحو مباشـر في عملية التصـنيف، فمثلاً تقانة آلة المتجه الداعم

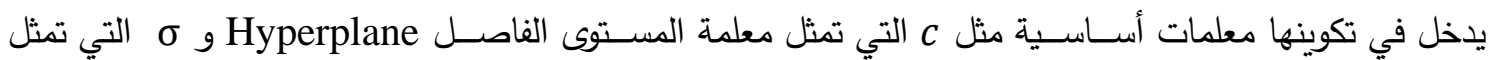
معلمة النواة Kernel ـ إذ تعطى قيم اختيارية وعثــوائية لهذه المعلمات أثناء بناء تقانة آلة المتجه الداعم وتركيبها، إلا أن اختيار هذه المعلمات بصورة عشوائية قد يؤدي إلى أخطاء في عملية التصنيف في كثير من الحالات إن لم تختر على نحو صحيح. إن عملية تصـــنيف البيانات في التطبيقات الطبية التي تعتمد على بيانات كبيرة مثل مرض اللوكيميا من النوعين سرطان الدم الليمفاوي الحاد Acute lymphocytic leukemia (ALL) وسـرطان الدم النخاعي الحاد Acute myeloid leukemia (AML) التقانة كونها تؤثر في عملية التصنيف على نحو مباشر نتيجة التعقيد الحاصل في طبيعة البيانات المستخدمة. وقد اقترح في هذه الدراسـة آلية تعتمد الخوارزمية الجينية في اختيار أفضـل قيم لمعلمات تقانة آلة المتجه الداعم مو و لتستخدم في بناء تركيب التقانة والحصول على امثل قيم المعلمات بالنسبة للبيانات المستخدمة [1].

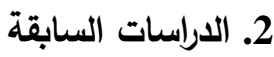

يركز في هذه الفقرة على أهم تفاصـيل الأعمال البحثية المختلفة المتعلقة بهذه الدراســة بخصــوص تقانتي

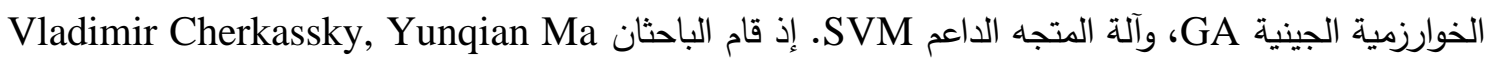

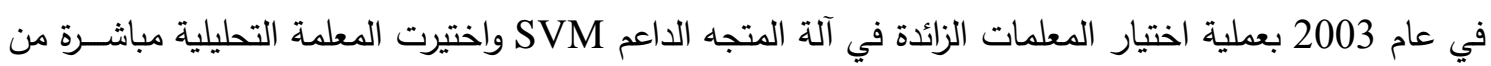

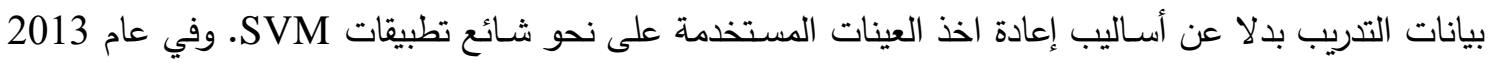

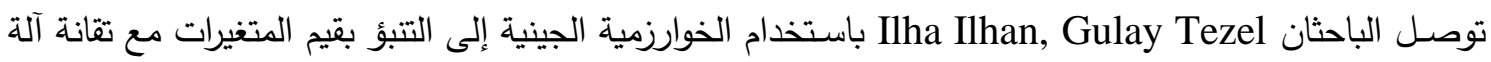

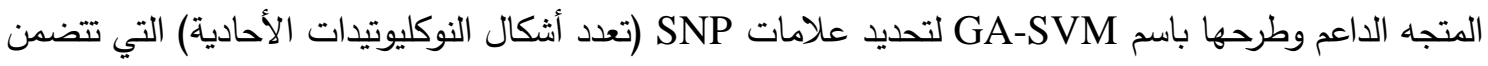
ملايين المتغيرات في الجينوم البثـري. إذ تم تحسين قيمة C معلمة آلة المتجه الداعم بوسـاطة الخوارزمية الجينية، وتظهر النتائج التي تم الحصـول عليها أن هذه الطريقة يمكن أن توفر أفضـل دقة في تحديد SNP مقارنة بالطرائق الأخرى.

\section{3. الخوارزمية الجينية}

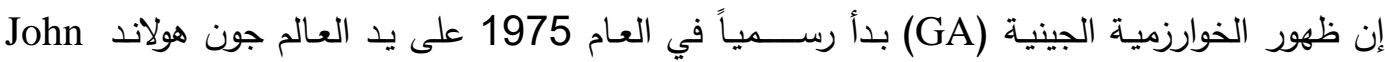

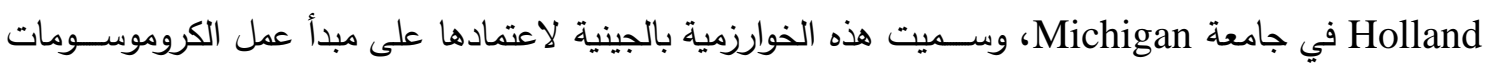

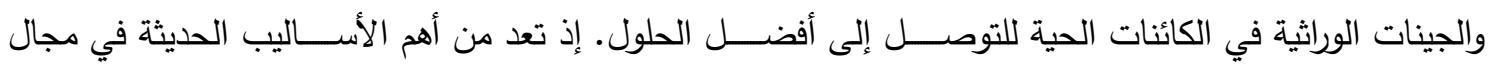

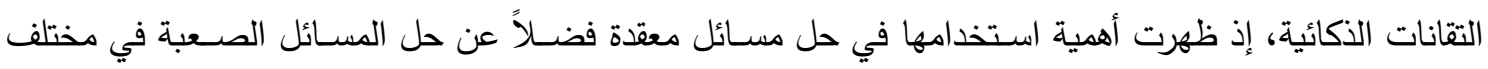

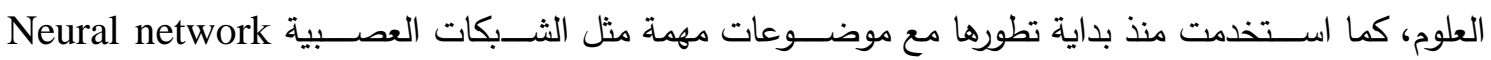


والروبوتات Optimization والامثلية مobotics، وكذلك حل مســائل التثـفير وكسـر الثــفرة وغيرها، إذ تهتم

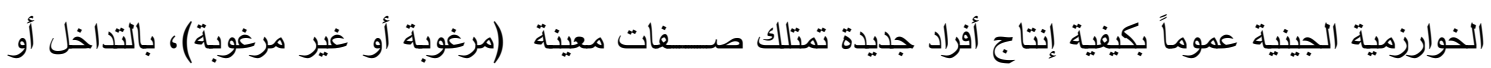
التعديل أو التبديل الذي يحصل على المجموعـات الموروثة بهدف تكوين هذه الأفراد، إذ ركز عليها الباحثون كثيراً،

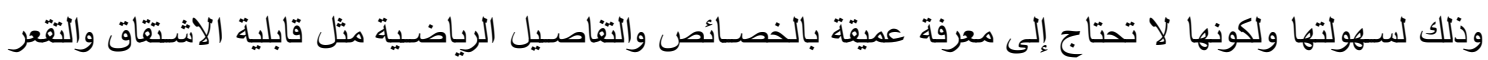

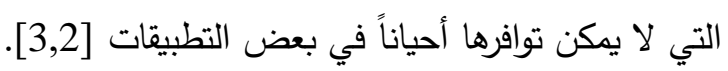

1.3. دالة اللياقة

تعد دالة اللياقة أهم المكونات الأساسية التي تعتمدها الخوارزمية الجينية، إذ تحسب فيها لياقة كل كروموسوم (فرد) في المجتمع، كما تسمى أحياناً بدالة الهدف للخوارزمية الجينية التي بها تحدد الأفراد التي يتم اعتمادها في حل

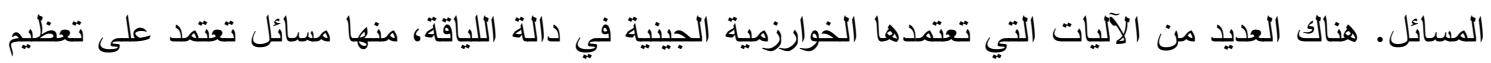
Maximization الإنتاج والربح، أما المسائل التي تعتمد على التصغير Minimization فإن الهدف منها هو إيجاد الحل ذي القيمة

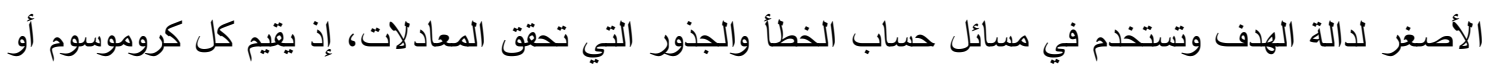

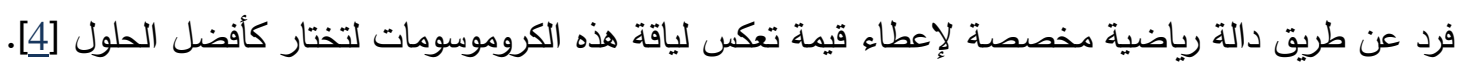

2.3

تعتمد آلية الخوارزمية الجينية على ثلاثة مراحل مهمة للمعالجة وهي [5, 1. الاختيار Selection. 2. 3. Mutation الطفرة الطزع.

Selection 1.2.3 الاختيار

هي عملية اختيار الزوج المناســب لكل كروموســوم من المجتمع الابتدائي، إذ يختار الأزواج لأجل التزاوج

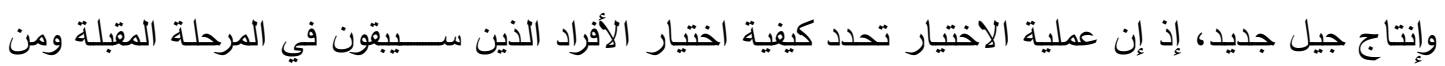
الأساليب المتبعة لاختيار الأزواج هي:

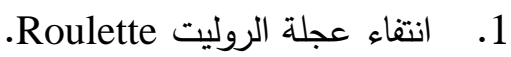

2. اختيار المجموعات Tournament.

3.

Crossover 2.2.3 التزاوج أو التقاطع

هي عملية إنتاج كروموسـومات جديدة ذات صـفات أفضـل من صـفات الأبوين ومن أهم أنواع طرائق التزاوج (التقاطع) في الخوارزمية الجينية:

1. تقاطع (تزاوج) ذو النقطة الواحدة Single point crossover.

2.

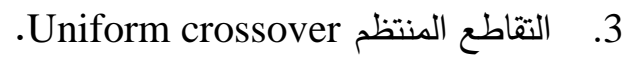


Mutation الطفرة 3.2.3

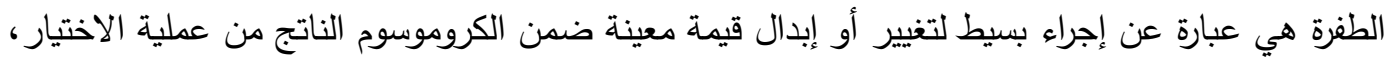
وإن القيمة المحددة المختارة لغرض إبدالها تختار عشوائياً، والطفرة عادة هي إجراء يجري على الفرد لغرض تحسين صفاته الجينية في المجتمع وهنالك عدة أنواع للطفرة ومنها:

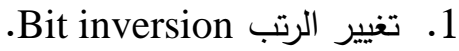

2. Adding or subtract الإضافة والطرح

3. مكس الجين Order changing.

3.3 مقياس التوقف

هنالك عدة مقاييس تعتمد في الخوارزمية الجينية لتحقيق شرط التوقف في حالة عدم الوصول للحل المثالي أو الأمثل، منها تتفيذ الخوارزمية عدد الأجيال المطلوبة ويحددها الباحثون، وكذلك تحديد وقت مســتغرق لتتفيذ الخوارزمية أو الحصـول على أحسن قيمة لدالة اللياقة في المجتمع الذي تم الوصـول إليه عندما تكون أقل من قيمة اللياقة التي حددت في حالة (Minimize) أو تتوقف عندما تكون أحســن قيمة لدالة اللياقة أكبر من قيمة اللياقة التي حددت في حالة (Maximize) ويفحص مقياس التوقف للخوارزمية الجينية بعد تكوين كل جيل جديد لنرى إذا

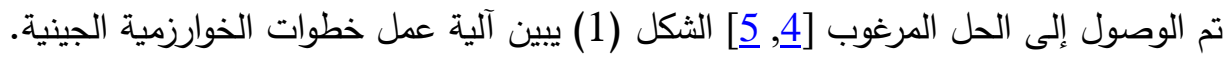

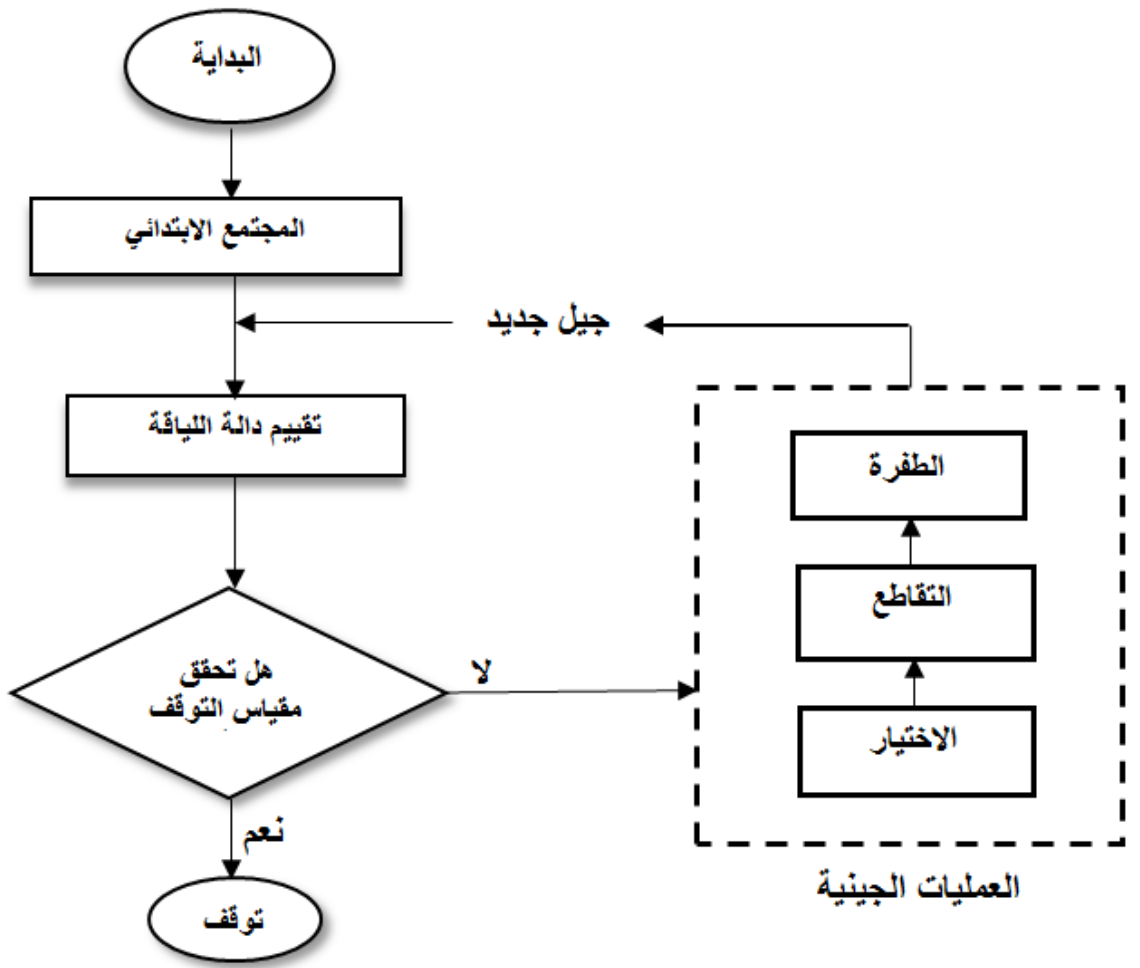

الثكل (1) يمثل مخططاً (نسيابياً يوضح خطوات عمل الخوارزمية الجينية 


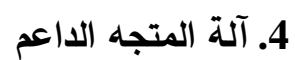

إن تقنات آلة المتجه الداعم SVM تعد من أهم التقانات المســتخدمة في تصــنيف البيانات، إذ تعتمد على

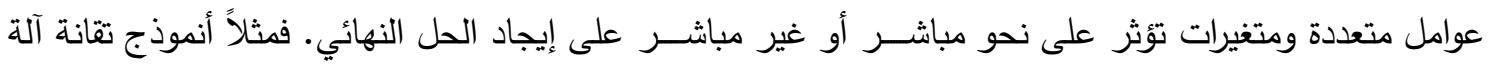

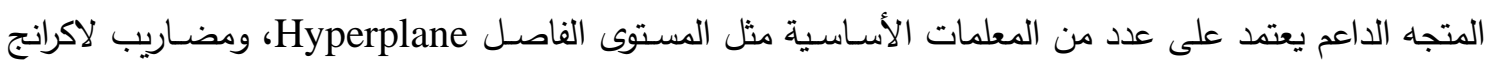
Lagrange multipliers فضاء الإدخال (Input Space)، تصنف على وفق الأنموذج الرياضي الآتي [4, 1] $\boldsymbol{w}^{T} \boldsymbol{x}_{i}+b \geq+1 \quad$ for $d_{i}=+1, \quad i=1,2, \ldots N$ $\boldsymbol{w}^{T} \boldsymbol{x}_{i}+b \leq-1 \quad$ for $d_{i}=-1, \quad i=1,2, \ldots N$

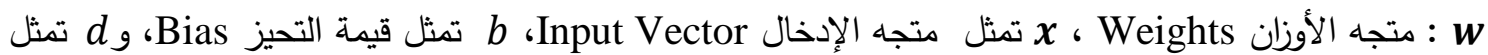
قيمة الإخراج. كما يمكن ملاحظة معادلات الحدود في المستوى من الثكل (2) الآتي:

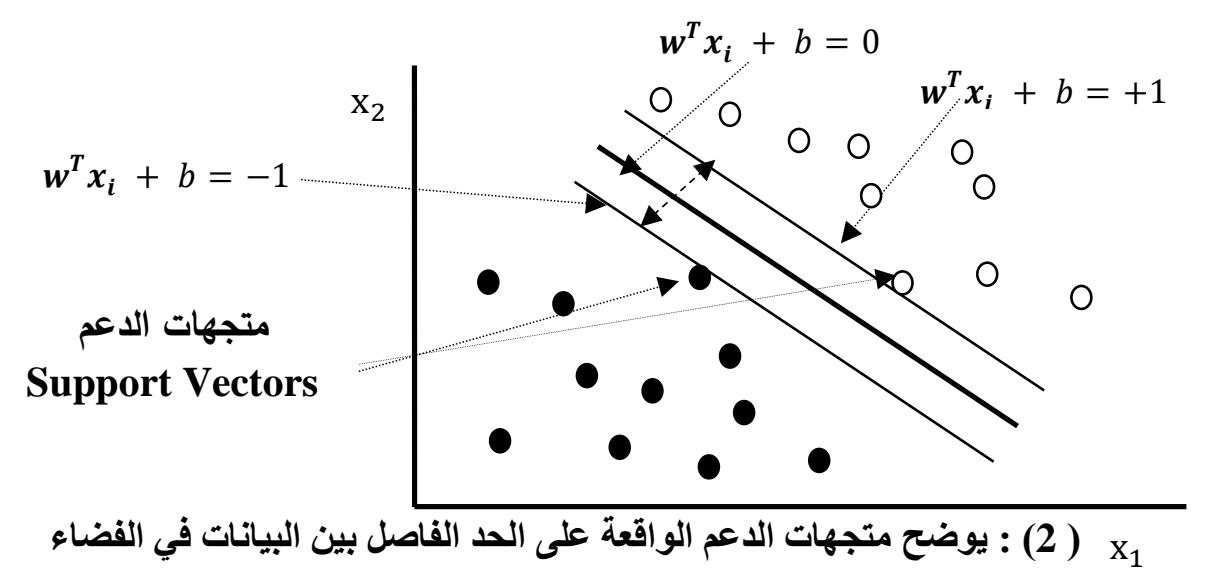

من الشكل (2) نلاحظ أن معادلة المستوى الفاصل Hyper plane تكتب بالثكل الآتي:

$\boldsymbol{w}^{T} \boldsymbol{x}_{i}+b=0$

إن البيانات القريبة أو التي تقع على حدود الحد الفاصل تسمى متجهات الدعم أو المساندة Support vectors. كما يمكن حساب المسافة بين النقاط في المستوى ومعادلة المستوى الفاصل من خلال العلاقة الآتية:

$d\left(\boldsymbol{w}, b, \boldsymbol{x}_{i}\right)=\frac{\left|\mathbf{w}^{\mathrm{T}} \mathbf{x}_{i}+\mathrm{b}\right|}{\|\mathbf{w}\|}$

وبعد عدد من الإجراءات والتحويلات الرياضية، يتم إيجاد قيم كل من متجه الأوزان المثالي (w*) والتحيز المثالي

$f(x)=I\left(\boldsymbol{w}^{*} \cdot \boldsymbol{x}+b^{*}\right)$

إذ إن $\boldsymbol{w}^{*}$ : تمثل الوزن المثالي ، l $^{*}$ : تمثل قيمة التحيز المثالية و sgn: تمثل القرار النهائي لانتماء (x) لأحد الأصناف [8] 1.4. عملية الفصل غير الخطي في الة المتجه الداعم 
إن عملية التصـنيف في أغلب التطبيقات تعتمد أسـلوب الفصـل غير الخطي Nonlinear لأن البيانات التي

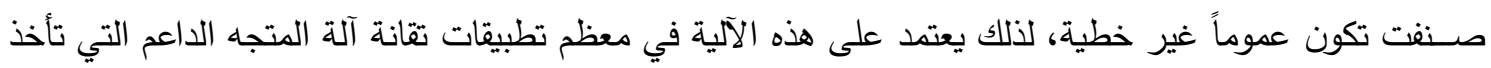

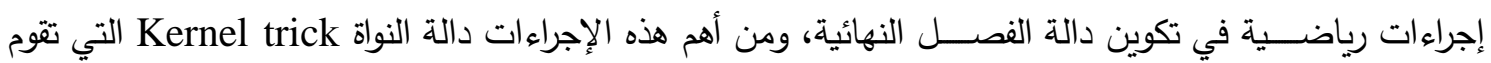
بتحويل البيانات من فضاء منخفض البعد Low dimensional إلى فضاء عالٍ البعد High dimensional من لاصدي أجل تصنيفها [10, 11,

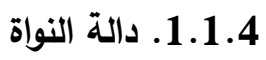
في بعض الحالات لا يمكن فصل البيانات بوساطة الحد الفاصل Hyperplane، لذلك يفضل استخدام تقانة

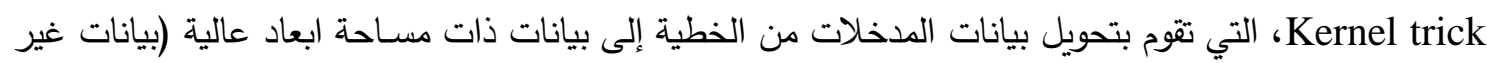

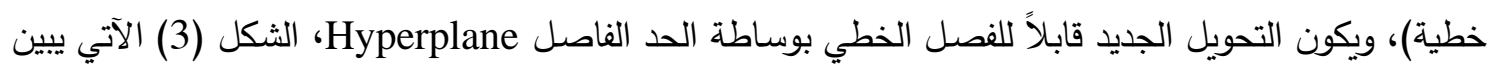
كيفية تحويل البيانات من الخطية إلى غير الخطية باستخدام

$\mathrm{T}: \mathrm{X} \longrightarrow \mathrm{Z}=\mathrm{T}(\mathrm{X})$

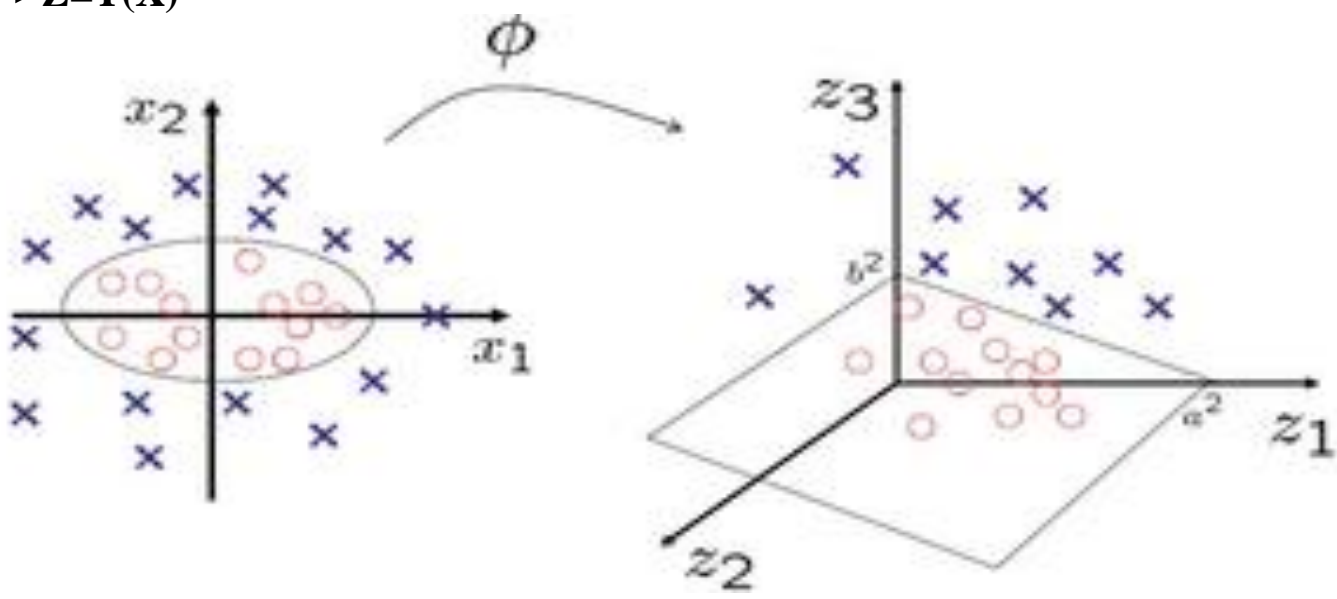

الثكل (3): يوضح عملية تحويل البيانات من الخطية إلى غير خطية باستخدام تقانة Kernel Trick

$T: X=\left(x_{1}, x_{2}, \ldots, x_{n)} \rightarrow Z=\left(z_{1}=T\left(x_{1}\right), z_{2}=T\left(x_{2}\right), \ldots, z_{n}=T\left(x_{n}\right)\right)\right.$.

2.1.4 أنواع دوال النواة

أهم أنواع دوال النواة التي تستخدم في مسائل التصنيف [12.13 13.

\begin{tabular}{|c|c|}
\hline iالدالة الصيغة الرياضية & \\
\hline Gaussian (RBF) kernel & $k\left(x_{i}, x_{j}\right)=\exp \left\langle-\frac{\left\|x_{i}-x_{j}\right\|^{2}}{2 \sigma^{2}}\right\rangle$ \\
\hline Polynomial kernel & $k\left(x_{i}, x_{j}\right)=\left(1+x_{i}^{t} x_{j}\right)^{d}$ \\
\hline Linear kernel & $k\left(x_{i}, x_{j}\right)=x_{i}^{t} x_{j}$ \\
\hline
\end{tabular}


3.1.4 مصائص دالة النواة

هناك العديد من الخصائص التي تعتمدها دالة النواة منها:

1. تكون متناظرة Symmetric:

$$
\begin{aligned}
& k(x, z)=(\varnothing(x) \cdot \emptyset(z))=(\varnothing(z) \cdot \varnothing(x))=k(z, x) \\
& \text { 2. تحقى شرط Cauchy-Schwarz Inequality: } \\
& k(x, z)^{2}=(\varnothing(x) \cdot \emptyset(z))^{2} \leq\|\varnothing(x)\|^{2}\|\varnothing(z)\|^{2} \\
& =(\varnothing(x) \cdot \varnothing(x))(\varnothing(z) \cdot \varnothing(z)) \\
& =k(x, x) k(z, z)
\end{aligned}
$$

Cross-Valind أسلوب الـ

يعد أسلوب الـــ Cross - Valind من أهم الأساليب المستخدمة في عملية تصنيف البيانات كونه يعطي آلية محايدة وذات كفاءة عالية في بناء المصنفات المختلفة، إذ يعتمد هذا الأسلوب على تقسيم البيانات إلى مجموعتين

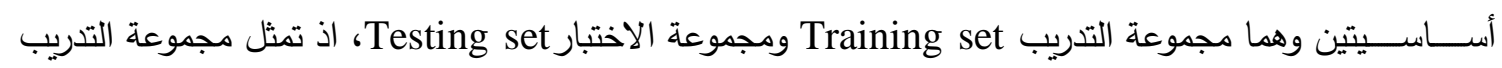
مجموعة البيانات التي سـوف تسـتخدم لغرض تكوين الأنموذج بينما مجموعة الاختبار تمثل مجموعة البيانات التي سوف تستخدم لغرض التنبؤ بالأنموذج الذي تم تكوينه من مجموعة بيانات التدريب، إذ يعتدد أسلوب ال - Cross

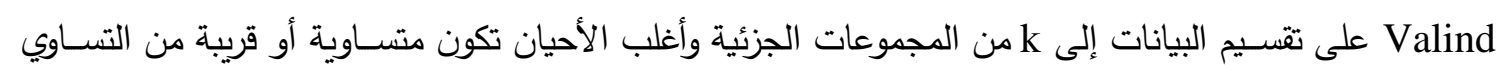

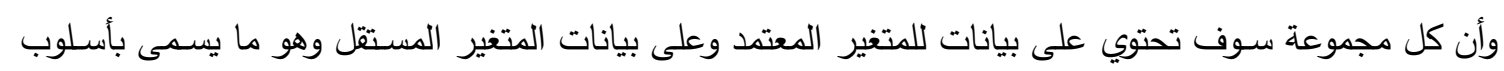

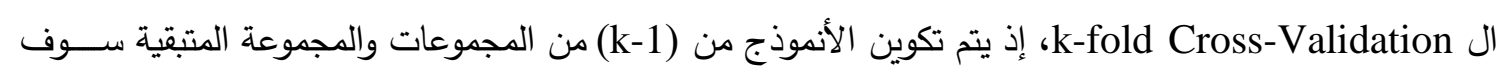

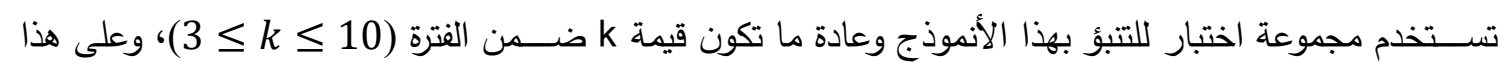

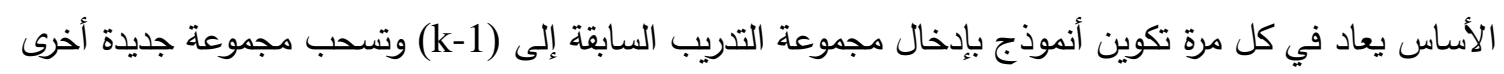

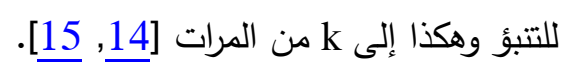

6A_SVM الطريقة المقترحة

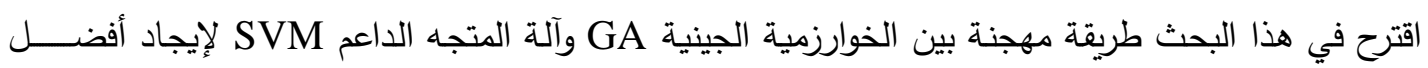

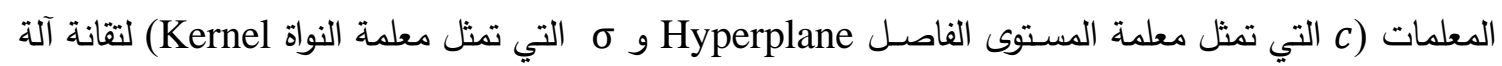

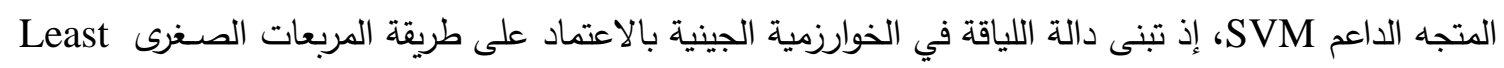

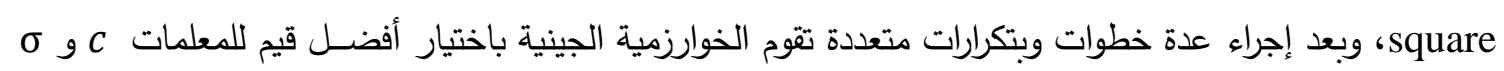

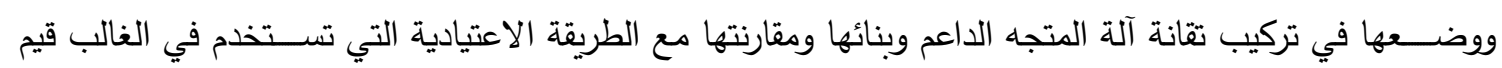
عشوائية للمعلمات C و م في في تركيب التقانة.

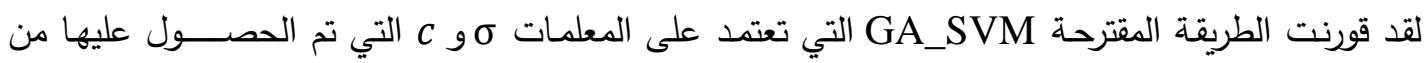

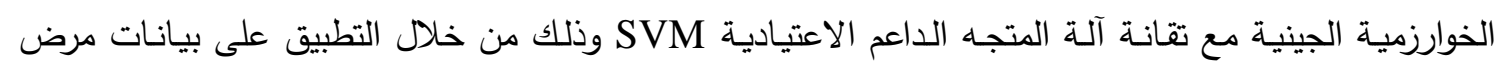

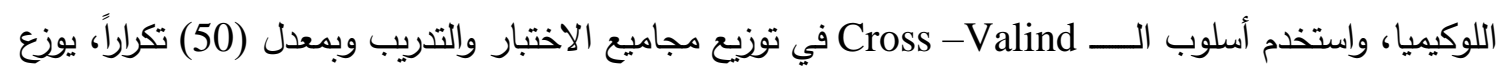

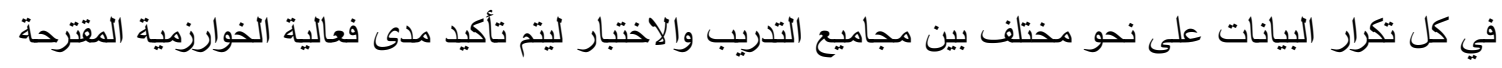




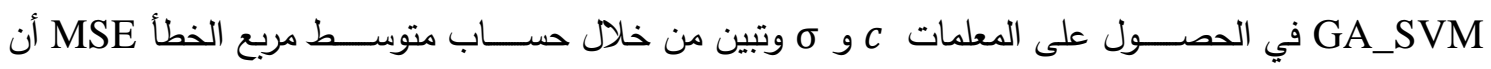

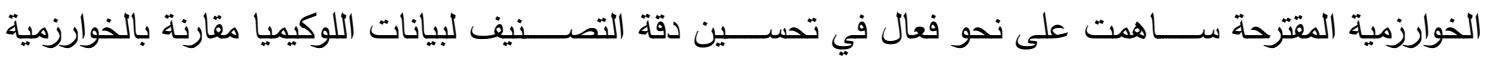
الاعتيادية.

\section{6. الخوارزمية المقترحة لتصنيف أمراض اللوكيميا ALL و وAML تكون على النحو الآتي:}

1- تهيئة بيانات الإدخال بثــكل مصـفوفة A صــفوفها تمثل عدد الحالات المرضــية الكلية وأعمدتها تمثل عدد

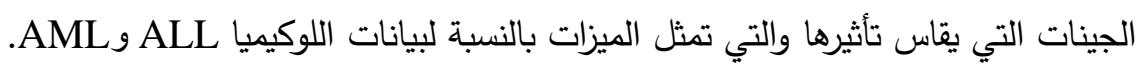

2- استخدام أسلوب ال Cross Valind لتقسيم البيانات المرضية إلى مجموعات تدريب Training وأخرى اختبار .Testing

3- استخدام الخوارزمية الجينية GA ومن خلال مفهوم دالة اللياقة في الحصول على أفضل المعلمات م و c. 4- اســتخدام أفضــل المعلمات م و c التي تم الحصــول عليها من الخوارزمية الجينية GA في تركيب تقانة آلة المتجه الداعم SVM وبنائها لأجل تصنيف بيانات التدريب والاختبار .

مقارنة نتائج الطريقة المقترحة GA_SVM التي بنيت بالاعتماد على أفضل المعلمات م و c مع تقانة آلة المتجه الداعم SVM الاعتيادية. 


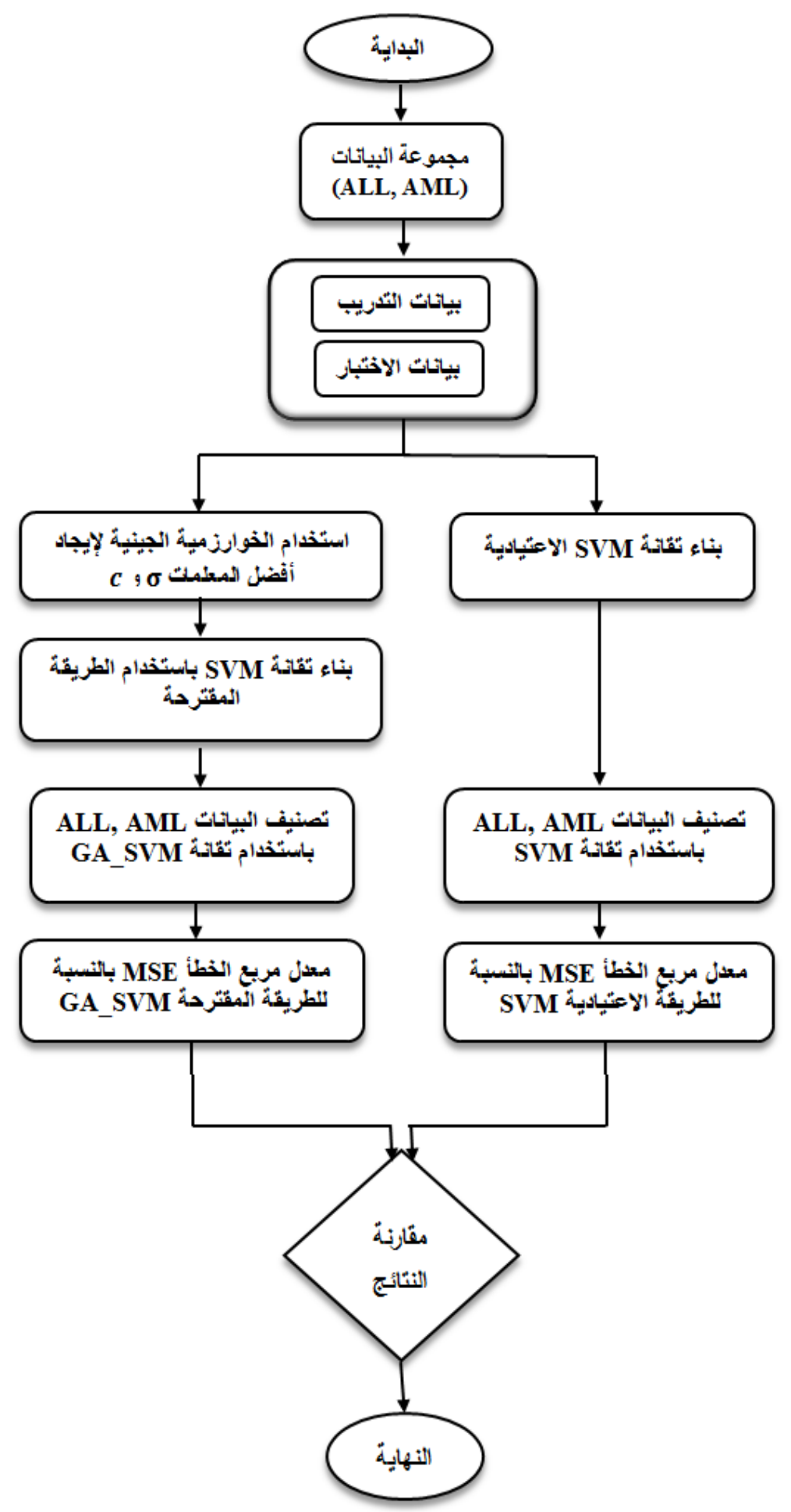

الثكل (4) مخطط يوضح آلية عمل الطريقة المقترحة

7.

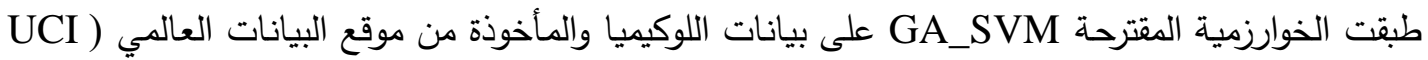
كachine Learning Repository نوعين (ALL, AML) وكل حالة تحتوي على 7129 ميزة (Feature) أو جين لكل حالة مرضــية، يعد كل منها قياس مدى فعالية جين معين وتمثل بأعداد حقيقية، تم الحصــــل على هذه الميزات عن طريق المصــفوفة الدقيقة 
Microarray. إذ استخدم أسلوب الــ Cross -Valind لتقسيم البيانات بصورة مختلفة (أي عندما تكون قيمة الـ K-fold=5 وعندما تكون قيمة الــ K-fold=10 وعندما تكون قيمة الــ K-fold=15) وذلك للتحقق من النتائج على نحو دقيق، إذ قورنت الطريقة المقترحة مع الطريقة الاعتيادية على النحو الآتي:

الجدول (1): يبين مقارنة بين تقانة SVM الاعتيادية والخوارزمية المقترحة GA_SVM عندما (k

(fold=5

\begin{tabular}{|c|c|c|}
\hline متوسط مربع الخطأ بالنسبة لبيانات & متوسط مربع الخطأ بالنسبة & التقانة المستخدمة \\
\hline $1.8333 e-02$ & $\mathbf{0}$ & الطريقة المقترحة \\
\hline 3.3833e- 02 & $\mathbf{0}$ & $\begin{array}{l}\text { الطريقة الاعتيادية } \\
\text { SVM }\end{array}$ \\
\hline
\end{tabular}

الجدول (2): يبين مقارنة بين تقانة SVM الاعتيادية والخوارزمية المقترحة GA_SVM عندما (k-)

(fold=10

\begin{tabular}{|c|c|c|}
\hline متوسط مربع الخطأ بالنسبة & متوسط مربع الذطأ بالنسبة & لتقانة المستخدمة \\
\hline $5.0000 \mathrm{e}-03$ & $\mathbf{0}$ & $\begin{array}{c}\text { الطريقة المقترحة } \\
\text { GA_SVM }\end{array}$ \\
\hline $1.5159 \mathrm{e}-02$ & $\mathbf{0}$ & $\begin{array}{c}\text { لطريقة الاعتيادية } \\
\text { SVM }\end{array}$ \\
\hline
\end{tabular}

الجدول (3): يبين مقارنة بين تقانة SVM الاعتيادية والخوارزمية المقترحة GA_SVM عندما (kAM

(fold=15

\begin{tabular}{|c|c|c|}
\hline متوسط مربع الخطأ بالنسبة & متوسط مربع الخطأ بالنسبة & التقانة المستخدمة \\
\hline $\mathbf{0}$ & $\mathbf{0}$ & $\begin{array}{c}\text { الطريقة المقترحة } \\
\text { GA_SVM }\end{array}$ \\
\hline $8.0000 \mathrm{e}-03$ & $4.0000 \mathrm{e}-03$ & $\begin{array}{l}\text { الطريقة الاعتيادية } \\
\text { SVM }\end{array}$ \\
\hline
\end{tabular}

اتضح من النتائج في الجداول (1) و (2) و (3) أعلاه أن الخوارزمية المقترحة GA_SVM تعطي نتائج أفضل من الخوارزمية الاعتيادية SVM في جميع الحالات التي اعتمدت كبيانات اختبار، في حين أن بيانات التدريب 
كانت مطابقة في الجداول الثلاثة للخوارزمية المقترحة، لكن الخوارزمية الاعتيادية اخطأت في بعض التصنيفات في الجدول (3)، مما يؤكد كفاءة الخوارزمية المقترحة وذلك بمقياس متوسط مربع الخطأ MSE.

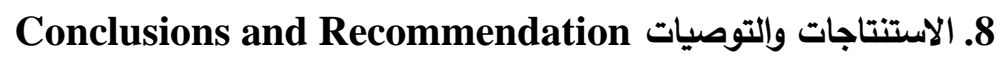

تضــنت هذه الدراسـة اقتراح الخوارزمية الجينية GA كطريقة لإيجاد افضـل قيم للمعلمات م و C في تقانة آلة

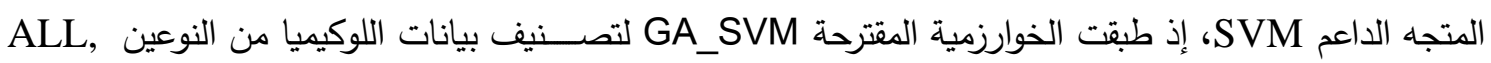

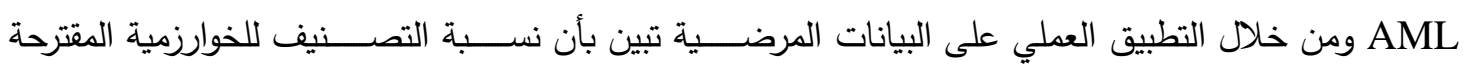
تقوق نسـبة التصـنيف لتقانة SVA_SVM

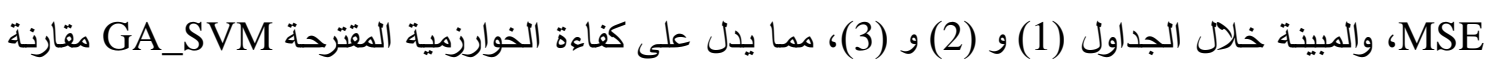
بتقانة آلة المتجه الداعم الاعتيادية. كما نوصي بدراسة تطوير تقانة آلة المتجه الداعم SVM بإيجاد أفضل المعلمات لها باستخدام التقانات الذكائية

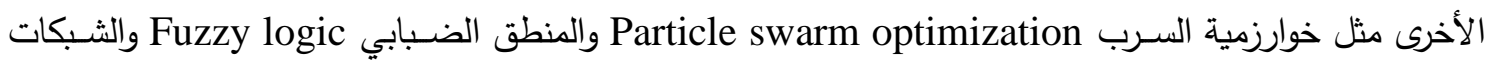

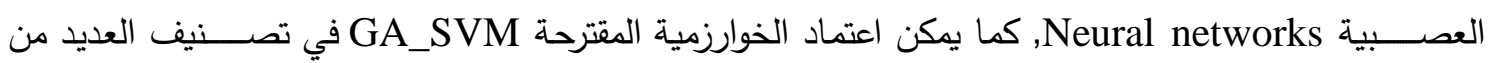
التطبيقات المختلفة التي تحتوي بيانات معقدة وكبيرة. 


\section{المصادر}

[1] Li, X.Z. and J.M. Kong, Application of GA-SVM method with parameter optimization for landslide development prediction. Natural Hazards and Earth System Science, 2014. 14(3): p. 525-533.

[2] Kozeny, V., Genetic algorithms for credit scoring: Alternative fitness function performance comparison. Expert Systems with Applications, 2015. 42(6): p. 2998-3004.

[3] Oreski, S. and G. Oreski, Genetic algorithm-based heuristic for feature selection in credit risk assessment. Expert Systems with Applications, 2014. 41(4): p. 20522064.

[4] Min, S.-H., J. Lee, and I. Han, Hybrid genetic algorithms and support vector machines for bankruptcy prediction. Expert Systems with Applications, 2006. 31(3): p. 652-660.

[5] Motieghader, H., et al., A hybrid gene selection algorithm for microarray cancer classification using genetic algorithm and learning automata. Informatics in Medicine Unlocked, 2017. 9: p. 246-254.

[6] Sastry, K., D.E. Goldberg, and G. Kendall, Genetic Algorithms. 2014: p. 93-117.

[7] Steinwart, I. and A. Christmann, Support vector machines. 2008: Springer Science $\&$ Business Media.

[8] Chen, F.-L. and F.-C. Li, Combination of feature selection approaches with SVM in credit scoring. Expert Systems with Applications, 2010. 37(7): p. 4902-4909.

[9] Zhou, L., K.K. Lai, and L. Yu, Credit scoring using support vector machines with direct search for parameters selection. Soft Computing, 2008. 13(2): p. 149-155.

[10] Bellotti, T. and J. Crook, Support vector machines for credit scoring and discovery of significant features. Expert Systems with Applications, 2009. 36(2): p. 33023308.

[11] Danenas, P. and G. Garsva, Selection of Support Vector Machines based classifiers for credit risk domain. Expert Systems with Applications, 2015. 42(6): p. 3194-3204.

[12] Huang, C.-L., M.-C. Chen, and C.-J. Wang, Credit scoring with a data mining approach based on support vector machines. Expert Systems with Applications, 2007. 33(4): p. 847-856.

[13] Shin, K.-S., T.S. Lee, and H.-j. Kim, An application of support vector machines in bankruptcy prediction model. Expert Systems with Applications, 2005. 28(1): p. 127-135.

[14] Maris, F., et al. Support vector Machines-Kernel algorithms for the estimation of the water supply in cyprus. in International Conference on Artificial Neural Networks. 2010. Springer.

[15] Shao, X. and M.-a. Sun, Predicting Gene Expression Noise from Gene Expression Variations, in Transcriptome Data Analysis. 2018, Springer. p. 183-198. 